\title{
Ulam Problem for the Sine Addition Formula in Hyperfunctions
}

\author{
by \\ Jaeyoung CHUNG and Dohan KIM
}

\begin{abstract}
We solve the Ulam problem for the sine addition formula in the spaces of Schwartz distributions and Gelfand hyperfunctions with respect to bounded distributions and bounded hyperfunctions.
\end{abstract}

2010 Mathematics Subject Classification: Primary 39B82; Secondary 46F99.

Keywords: convolution, distribution, hyperfunction, heat kernel, sine addition formula, Ulam problem.

\section{§1. Introduction}

In 1950, Laurent Schwartz introduced the theory of distributions in his monograph Théorie des distributions [33]. In this book Schwartz systematizes the theory of generalized functions, basing it on the theory of linear topological spaces, relates all the earlier approaches, and obtains many important results. After his elegant theory appeared, many important concepts and results on the classical spaces of functions have been generalized to the space of distributions. For example, positive functions and positive-definite functions have been generalized to positive distributions and positive-definite distributions, respectively, and it was shown that every positive distribution is a positive measure [22, p. 38] and every positivedefinite distribution is the Fourier transform of a positive measure $\mu$ such that $\int(1+|x|)^{-p} d \mu<\infty$ for some $p \geq 0$ [21, p. 157], which is called the BochnerSchwartz theorem and is a natural generalization of the famous Bochner theorem

Communicated by H. Okamoto. Received May 26, 2013. Revised August 22, 2013, and September $21,2013$.

J. Chung: Department of Mathematics, Kunsan National University,

Kunsan 573-701, Republic of Korea;

e-mail: jychung@kunsan.ac.kr

D. Kim: Department of Mathematics, Seoul National University,

Seoul 151-747, Republic of Korea;

e-mail: dhkim@snu.ac.kr

(c) 2014 Research Institute for Mathematical Sciences, Kyoto University. All rights reserved. 
stating that every positive-definite function is the Fourier transform of a positive finite measure. For other examples, the Paley-Wiener theorem has been generalized to the Paley-Wiener-Schwartz theorem which characterizes the distributions with bounded supports [22, p. 181].

The main purpose of this paper is to prove the Hyers-Ulam type stability for the sine functional equation

$$
f(x+y)-f(x) g(y)-g(x) f(y)=0
$$

in Schwartz distributions and Gelfand hyperfunctions. The Ulam problem for functional equations goes back to 1940 when S. M. Ulam proposed the following [36]:

Let $f$ be a mapping from a group $G_{1}$ to a metric group $G_{2}$ with metric $d(\cdot, \cdot)$ such that

$$
d(f(x y), f(x) f(y)) \leq \epsilon \quad \text { for all } x, y \in G_{1} .
$$

Then does there exist a group homomorphism $h$ and $\delta_{\epsilon}>0$ such that

$$
d(f(x), h(x)) \leq \delta_{\epsilon} \quad \text { for all } x \in G_{1} ?
$$

This problem was solved affirmatively by D. H. Hyers under the assumption that $G_{2}$ is a Banach space (see Hyers [23], Hyers-Isac-Rassias [24]). In 19491951, this result was generalized by T. Aoki [2] and D. G. Bourgin [6, 7]. Since then Ulam problems for many other functional equations have been investigated [17-19, 25, 27-32]. Among the many results obtained, L. Székelyhidi developed an idea of using invariant subspaces of functions defined on a group or semigroup in connection with the Ulam problem for sine functional equations [34,35]. As a direct consequence of the elegant results of Székelyhidi, it was shown that if $f, g: \mathbb{R}^{n} \rightarrow \mathbb{C}$ satisfy

$$
|f(x+y)-f(x) g(y)-g(x) f(y)| \leq M, \quad x, y \in \mathbb{R}^{n},
$$

for some $M>0$, then either there exist $\lambda_{1}, \lambda_{2} \in \mathbb{C}$, not both zero, and $N>0$ such that

$$
\left|\lambda_{1} f(x)-\lambda_{2} g(x)\right| \leq N
$$

for all $x \in \mathbb{R}^{n}$, or else

$$
f(x+y)-f(x) g(y)-g(x) f(y)=0
$$

for all $x, y \in \mathbb{R}^{n}$. Furthermore, the functions $f$ and $g$ satisfying both (1.1) and (1.2) were investigated.

As a generalization of (1.1), it is very natural to consider

$$
f(x+y)-f(x) g(y)-g(x) f(y) \in L^{\infty}\left(\mathbb{R}^{2 n}\right),
$$


where $f$ and $g$ are Lebesgue measurable functions and $L^{\infty}\left(\mathbb{R}^{2 n}\right)$ is the space of all bounded measurable functions defined in $\mathbb{R}^{2 n}$. Note that (1.4) means that the inequality (1.1) holds almost everywhere. In [8-12], some stability problems for several functional equations including the condition (1.4) were considered in various spaces of generalized functions including Schwartz distributions. In [10-12], for example, replacing $f$ and $g$ by distributions $u$ and $v$ in (1.4) we have considered the condition

$$
u \circ S-u \otimes v-v \otimes u \in L^{\infty}\left(\mathbb{R}^{2 n}\right),
$$

where $S(x, y)=x+y, x, y \in \mathbb{R}^{n}$, and $\circ$ and $\otimes$ denote the pullback and the tensor product of generalized functions, respectively. The condition (1.5) is not formulated purely in the language of generalized functions because the differences are assumed to be classical bounded measurable functions; all the previous results in [10-12] have formulations as in (1.5).

Schwartz [33] generalized the space $L^{\infty}\left(\mathbb{R}^{n}\right)$ of bounded measurable functions to the space $\mathcal{D}_{L^{\infty}}^{\prime}\left(\mathbb{R}^{n}\right)$ of bounded distributions. Taking this into account, it is natural to consider the following stability condition for the sine functional equation in distributions and hyperfunctions $u, v$ with respect to bounded distributions and bounded hyperfunctions:

$$
u \circ S-u \otimes v-v \otimes u \in \mathcal{D}_{L^{\infty}}^{\prime}\left(\mathbb{R}^{2 n}\right)\left[\operatorname{resp} . \mathcal{A}_{L^{\infty}}^{\prime}\left(\mathbb{R}^{2 n}\right)\right],
$$

where $\mathcal{D}_{L^{\infty}}^{\prime}\left(\mathbb{R}^{2 n}\right)$ and $\mathcal{A}_{L^{\infty}}^{\prime}\left(\mathbb{R}^{2 n}\right)$ are the spaces of bounded distributions and bounded hyperfunctions respectively, and $S, \circ, \otimes$ are as in (1.5). For some related results in Schwartz distributions, we refer the reader to [3-5, 8,9,22,33]. The main tools of our proof are based on structure theorems for generalized functions (see Lemmas 4.3 and 4.4 below) and the heat kernel method initiated by T. Matsuzawa [26], which represents generalized functions as initial values of solutions of the heat equation with appropriate growth conditions [13-16,26] (see Lemmas 4.1 and 4.2). Making use of the heat kernel method we convert (1.6) to the following classical stability statement: there exist $C, N, d>0$ [resp. for every $\epsilon>0$ there exist $C_{\epsilon}>0$ ] such that

$$
\begin{aligned}
\mid \tilde{u}(x+y, t+s)-\tilde{u}(x, t) \tilde{v}(y & , s)-\tilde{v}(x, t) \tilde{u}(y, s) \mid \\
& \leq C\left(\frac{1}{t}+\frac{1}{s}\right)^{N}+d\left[\text { resp. } C_{\epsilon} e^{\epsilon(1 / t+1 / s)}\right]
\end{aligned}
$$

for all $x, y \in \mathbb{R}^{n}$ and $t, s>0$, where $\tilde{u}, \tilde{v}: \mathbb{R}^{n} \times(0, \infty) \rightarrow \mathbb{C}$ are the solutions of the heat equation corresponding to $u, v$ respectively, which are introduced in Section 4. In Section 2, we consider the stability (1.7) and combining this result 
with the heat kernel method we prove the stability (1.6) in Section 4. Also, as direct consequences of our result we obtain the stability (1.5) and the following $L^{\infty}$-version of the stability for the sine functional equation:

$$
\|f(x+y)-f(x) g(y)-g(x) f(y)\|_{L^{\infty}\left(\mathbb{R}^{2 n}\right)} \leq C,
$$

where $f, g: \mathbb{R}^{n} \rightarrow \mathbb{C}$ are Lebesgue measurable functions satisfying the following growth condition: for every $\epsilon>0$ there exists $C_{\epsilon}>0$ such that

$$
|f(x)| \leq C_{\epsilon} e^{\epsilon|x|^{2}}
$$

for all $x \in \mathbb{R}^{n}$.

\section{$\S 2$. Stability problem in the classical sense}

Let $f, g: G \times(0, \infty) \rightarrow \mathbb{C}$ with $\langle G,+\rangle$ an Abelian group. Throughout this paper $N$ denotes a fixed nonnegative real number. We consider the following stability statements involving functional inequalities:

There exist $C>0$ and $d>0$ such that

$$
\begin{aligned}
& |f(x+y, t+s)-f(x, t) g(y, s)-g(x, t) f(y, s)| \leq C\left(\frac{1}{t}+\frac{1}{s}\right)^{N}+d \\
& \qquad \text { for all } x, y \in G \text { and } t, s>0
\end{aligned}
$$

for every $\epsilon>0$ there exists $C_{\epsilon}>0$ such that

$$
\begin{gathered}
|f(x+y, t+s)-f(x, t) g(y, s)-g(x, t) f(y, s)| \leq C_{\epsilon} e^{\epsilon(1 / t+1 / s)} \\
\text { for all } x, y \in G \text { and } t, s>0 .
\end{gathered}
$$

From now on, a function $A$ from a semigroup $\langle S,+\rangle$ to the field $\mathbb{C}$ of complex numbers is said to be additive if $A(x+y)=A(x)+A(y)$ for all $x, y \in S$, and $m: S \rightarrow \mathbb{C}$ is said to be an exponential function provided $m(x+y)=m(x) m(y)$ for all $x, y \in S$.

We introduce the following conditions on $f: G \times(0, \infty) \rightarrow \mathbb{C}$ and $N$ :

$$
\text { There exist } C>0 \text { and } d>0 \text { such that }
$$

$$
|f(x, t)| \leq C t^{-N}+d, \quad \forall x \in G, t>0 ;
$$

for every $\epsilon>0$, there exists $C_{\epsilon}>0$ such that

$$
|f(x, t)| \leq C_{\epsilon} e^{\epsilon / t}, \quad \forall x \in G, t>0 .
$$


Lemma 2.1 ([1, p. 212]). All solutions $f, g: G \times(0, \infty) \rightarrow \mathbb{C}$ of the functional equation

$$
f(x+y, t+s)-f(x, t) g(y, s)-g(x, t) f(y, s)=0, \quad x, y \in G, t, s>0,
$$

are given by one of the following:

(i) $f=0$ and $g$ is arbitrary,

(ii) $f(x, t)=\frac{1}{2 \lambda} m(x, t)$ and $g(x, t)=\frac{1}{2} m(x, t)$, where $\lambda \in \mathbb{C}$ and $m$ is a nonzero exponential function,

(iii) $g(x, t)=m(x, t)$ and $f(x, t)=A(x, t) m(x, t)$, where $A$ is a nonzero additive function and $m$ is a nonzero exponential function,

(iv) $f(x, t)=\frac{1}{2 \lambda}\left(m^{*}(x, t)-m^{* *}(x, t)\right)$ and $g(x, t)=\frac{1}{2}\left(m^{*}(x, t)+m^{* *}(x, t)\right)$, where $\lambda \in \mathbb{C}$ and $m^{*}, m^{* *}$ are nonzero exponential functions.

Using the idea in [24, p. 104] we obtain the following.

Lemma 2.2. Let $f, g: G \times(0, \infty) \rightarrow \mathbb{C}$ satisfy the following condition for some $N \geq 0:$ for each $y \in G$ and $s>0$ there exist positive constants $C=C(y, s)$ and $d=d(y, s)[$ resp. for each $y \in G, s>0$ and $\epsilon>0$ there exists a positive constant $\left.C_{\epsilon}=C_{\epsilon}(y, s)\right]$ such that

$$
|f(x+y, t+s)-f(x, t) g(y, s)| \leq C t^{-N}+d\left[\text { resp. } C_{\epsilon} e^{\epsilon / t}\right]
$$

for all $x \in G$ and $t>0$. Then either $f$ satisfies (2.3) [resp. (2.4)] or $g$ is an exponential function.

Proof. Suppose that $g$ is not exponential. Then there exist $y_{0}, z_{0} \in G$ and $s_{0}, r_{0}>0$ such that $g\left(y_{0}+z_{0}, s_{0}+r_{0}\right) \neq g\left(y_{0}, s_{0}\right) g\left(z_{0}, r_{0}\right)$. Now, we can write

$$
\begin{aligned}
f\left(x+y_{0}+z_{0}, t+\right. & \left.s_{0}+r_{0}\right)-f\left(x+y_{0}, t+s_{0}\right) g\left(z_{0}, r_{0}\right) \\
= & f\left(x+y_{0}+z_{0}, t+s_{0}+r_{0}\right)-f(x, t) g\left(y_{0}+z_{0}, s_{0}+r_{0}\right) \\
& -g\left(z_{0}, r_{0}\right)\left(f\left(x+y_{0}, t+s_{0}\right)-f(x, t) g\left(y_{0}, s_{0}\right)\right) \\
& +f(x, t)\left(g\left(y_{0}+z_{0}, s_{0}+r_{0}\right)-g\left(y_{0}, s_{0}\right) g\left(z_{0}, r_{0}\right)\right),
\end{aligned}
$$

and hence

$$
\begin{aligned}
f(x, t)=( & \left.g\left(y_{0}+z_{0}, s_{0}+r_{0}\right)-g\left(y_{0}, s_{0}\right) g\left(z_{0}, r_{0}\right)\right)^{-1} \\
\times & \left(f\left(x+y_{0}+z_{0}, t+s_{0}+r_{0}\right)-f\left(x+y_{0}, t+s_{0}\right) g\left(z_{0}, r_{0}\right)\right. \\
& \quad-f\left(x+y_{0}+z_{0}, t+s_{0}+r_{0}\right)+f(x, t) g\left(y_{0}+z_{0}, s_{0}+r_{0}\right) \\
& \left.\quad+g\left(z_{0}, r_{0}\right)\left(f\left(x+y_{0}, t+s_{0}\right)-f(x, t) g\left(y_{0}, s_{0}\right)\right)\right) .
\end{aligned}
$$


It follows from (2.6) and (2.7) that there exist positive constants $C_{1}, C_{2}, C_{3}, d_{1}, d_{2}$, $d_{3}, C^{\prime}$ and $d^{\prime}$ [resp. for every $\epsilon>0$ there exists a positive constant $C_{\epsilon}^{\prime}$ ] such that

$$
\begin{aligned}
|f(x, t)| & \leq C_{1}\left(t+s_{0}\right)^{-N}+d_{1}+C_{2} t^{-N}+d_{2}+C_{3} t^{-N}+d_{3} \\
& \leq C^{\prime} t^{-N}+d^{\prime}\left[\text { resp. } C_{\epsilon}^{\prime} e^{\epsilon / t}\right]
\end{aligned}
$$

for all $x \in G$ and $t>0$.

Lemma 2.3. Let $g: G \times(0, \infty) \rightarrow \mathbb{C}$ be a nonzero exponential function satisfying (2.3) [resp. (2.4)]. Then $g$ can be written in the form

$$
g(x, t)=m_{1}(x) m_{2}(t),
$$

where $m_{1}$ is an exponential function on $G$ satisfying $\left|m_{1}(x)\right|=1$ for all $x \in G$ and $m_{2}$ is an exponential function on $(0, \infty)$ satisfying $0<\left|m_{2}(t)\right| \leq 1$ for all $t>0$.

Proof. Assume that $g\left(x_{0}, t_{0}\right)=0$ for some $x_{0} \in G$ and $t_{0}>0$. Then, for given $x \in G$ and $t>0$, choosing a positive integer $k$ such that $k t>t_{0}$ we have

$$
[g(x, t)]^{k}=g(k x, k t)=g\left(k x-x_{0}, k t-t_{0}\right) g\left(x_{0}, t_{0}\right)=0 .
$$

Thus, $g(x, t) \neq 0$ for all $x \in G$ and $t>0$. Let

$$
m(x, t)=g(x, t) g(0, t)^{-1} .
$$

Then

$$
\begin{aligned}
m(x, t) & =g(x, t) g(0, t)^{-1}=g(x, t+s) g(0, s)^{-1} g(0, t)^{-1}=g(x, s) g(0, s)^{-1} \\
& =m(x, s) .
\end{aligned}
$$

Hence, $m$ is independent of $t>0$ and we can write $m(x, t)=: m_{1}(x)$. Now,

$m_{1}(x+y)=g(x+y, 2 t) g(0,2 t)^{-1}=m(x, t) g(0, t)^{-1} g(y, t) g(0, t)^{-1}=m_{1}(x) m_{1}(y)$.

Thus, $m_{1}$ is an exponential function and we can write

$$
g(x, t)=m_{1}(x) g(0, t)=: m_{1}(x) m_{2}(t),
$$

where $m_{1}$ is an exponential function on $G$ and $m_{2}$ is an exponential function on $(0, \infty)$. It follows from $(2.3)$ [resp. (2.4)] that $m_{1}$ is bounded and $m_{2}$ satisfies

$$
\left|m_{2}(t)\right| \leq C t^{-N}+d\left[\text { resp. } C_{\epsilon} e^{\epsilon / t}\right]
$$

for all $t>0$. If there exists $x_{0} \in G$ such that $\left|m_{1}\left(x_{0}\right)\right|>1$ or $\left|m_{1}\left(x_{0}\right)\right|<1$, then for all integers $k$ we have $\left|m_{1}\left(k x_{0}\right)\right|=\left|m_{1}\left(x_{0}\right)\right|^{k} \rightarrow \infty$ as $k \rightarrow \infty$ or $k \rightarrow-\infty$. Thus, $\left|m_{1}(x)\right|=1$ for all $x \in G$. Similarly, if $\left|m_{2}\left(t_{0}\right)\right|>1$ for some $t_{0}>0$, then for all integers $k$ we have $\left|m_{2}\left(k t_{0}\right)\right|=\left|m_{2}\left(t_{0}\right)\right|^{k} \rightarrow \infty$ as $k \rightarrow \infty$, which contradicts the inequality (2.8). 
Lemma 2.4. Let $g$ be a nonzero exponential function satisfying (2.3) [resp. (2.4)]. Suppose that $f, g: G \times(0, \infty) \rightarrow \mathbb{C}$ satisfy (2.1) [resp. (2.2)]. Then

$$
\begin{aligned}
& g(x, t)=m_{1}(x) m_{2}(t), \\
& f(x, t)=l(x) m_{1}(x) m_{2}(t)+2 f(0, t / 2) g(0, t / 2) m_{1}(x)+R(x, t),
\end{aligned}
$$

where $m_{1}$ and $m_{2}$ are exponential functions on $G$ and $(0, \infty)$ respectively such that $\left|m_{1}(x)\right|=1$ for all $x \in G$ and $0<\left|m_{2}(t)\right| \leq 1$ for all $t>0, l(x)$ is an additive function on $G$, and $R$ is a function satisfying (2.3) [resp. (2.4)].

Proof. Dividing both sides of (2.1) [resp. (2.2)] by $g(x+y, t+s)$, setting $h(x, t)=$ $f(x, t) g(x, t)^{-1}$ and using Lemma 2.3 we have

$$
\begin{aligned}
\mid h(x+y, t+s)-h(x, t) & -h(y, s) \mid \\
\leq & \left(C\left(\frac{1}{t}+\frac{1}{s}\right)^{N}+d\right)\left|m_{1}(x) m_{1}(y) m_{2}(t) m_{2}(s)\right|^{-1} \\
\leq & \left(C\left(\frac{1}{t}+\frac{1}{s}\right)^{N}+d\right)\left|m_{2}(t) m_{2}(s)\right|^{-1} \\
& {\left[\operatorname{resp} . C_{\epsilon} e^{\epsilon(1 / t+1 / s)}\left|m_{2}(t) m_{2}(s)\right|^{-1}\right] }
\end{aligned}
$$

for all $x, y \in G$ and $t, s>0$. Thus,

$$
|h(x+y, t+s)-h(x, t)-h(y, s)| \leq \psi(t, s)
$$

for all $x, y \in G$ and $t, s>0$, where

$$
\begin{aligned}
\psi(t, s)= & \left(C\left(\frac{1}{t}+\frac{1}{s}\right)^{N}+d\right)\left|m_{2}(t) m_{2}(s)\right|^{-1} \\
& {\left[\text { resp. } C_{\epsilon} e^{\epsilon(1 / t+1 / s)}\left|m_{2}(t) m_{2}(s)\right|^{-1}\right] . }
\end{aligned}
$$

Replacing $s$ by $t$ and putting $y=0$ in (2.9) we obtain

$$
|h(x, 2 t)-h(x, t)-h(0, t)| \leq \psi(t, t)
$$

for all $x \in G$ and $t>0$. Replacing $t$ by $s$ and putting $x=0$ in (2.9) we have

$$
|h(y, 2 s)-h(0, s)-h(y, s)| \leq \psi(s, s)
$$

for all $y \in G$ and $s>0$. Using the triangle inequality together with (2.9), (2.11) and (2.12) we find that

$$
\begin{aligned}
\mid h(x+y, t+s)-h(x, 2 t)-h(y, 2 s)+ & h(0, t)+h(0, s) \mid \\
& \leq \psi(t, s)+\psi(t, t)+\psi(s, s)
\end{aligned}
$$


for all $x, y \in G$ and $t, s>0$. Replacing $y$ by $x$ and $s$ by $t$ in (2.13) yields

$$
|h(2 x, 2 t)-2 h(x, 2 t)+2 h(0, t)| \leq 3 \psi(t, t)
$$

for all $x \in G$ and $t>0$. Fixing $t>0$, replacing $x$ by $2^{k-1} x$ in (2.14) and dividing the result by $2^{k}$ we have

$$
\left|2^{-k} h\left(2^{k} x, 2 t\right)-2^{-k+1} h\left(2^{k-1} x, 2 t\right)+2^{-k+1} h(0, t)\right| \leq 3 \cdot 2^{-k} \psi(t, t)
$$

for all $x \in G$. For given positive integers $n, m$, putting $k=n, n+1, \ldots, n+m$ in (2.15), summing up the results and using the triangle inequality, we can see that $A_{n}(x, t):=2^{-n} h\left(2^{n} x, 2 t\right), n=1,2, \ldots$, is a Cauchy sequence and $A(x, t):=$ $\lim _{n \rightarrow \infty} A_{n}(x, t)$ exists. Replacing $x, y$ by $2^{n} x, 2^{n} y$ respectively in (2.13), dividing by $2^{n}$ and letting $n \rightarrow \infty$ we have

$$
A(x+y, t+s)-A(x, 2 t)-A(y, 2 s)=0
$$

for all $x, y \in G$ and $t, s>0$. Letting $x=y=0$ and replacing $t, s$ by $t / 2$ in $(2.16)$ we get $A(0, t)=0$ for all $t>0$. Thus, putting $y=0$ in (2.16) we have

$$
A(x, 2 t)=A(x, t+s)=A(x, s+t)=A(x, 2 s)
$$

for all $x \in G$ and $t, s>0$. It follows from (2.17) that $A(x, t)$ is independent of $t>0$ and is an additive function of $x \in G$, which we denote by $l(x)$. Using the triangle inequality together with $(2.15)$ for $k=1, \ldots, n$ and letting $n \rightarrow \infty$ gives

$$
|l(x)-h(x, 2 t)+2 h(0, t)| \leq 3 \psi(t, t)
$$

for all $x \in G$ and $t>0$. Replacing $t$ by $t / 2$ in (2.18) and multiplying the result by $|g(x, t)|$ we obtain

$$
\begin{aligned}
\mid f(x, t)-l & (x) g(x, t)-2 f(0, t / 2) g(0, t / 2) m_{1}(x) \mid \\
& \leq 3 C\left(4^{N} t^{-N}+d\right)\left|m_{2}(t / 2)\right|^{-2}|g(x, t)|=\left(C^{\prime} t^{-N}+d^{\prime}\right)|g(0, t)|^{-1}|g(x, t)| \\
& =\left(C^{\prime} t^{-N}+d^{\prime}\right)\left|m_{1}(x)\right|^{-1}=C^{\prime} t^{-N}+d^{\prime}\left[\operatorname{resp} . C_{\epsilon}^{\prime} e^{4 \epsilon / t}\right] .
\end{aligned}
$$

Letting $R(x, t):=f(x, t)-l(x) g(x, t)-2 f(0, t / 2) g(0, t / 2) m_{1}(x)$ we complete the proof.

Lemma 2.5. Suppose that $f, g: G \times(0, \infty) \rightarrow \mathbb{C}$ satisfy $(2.1)[$ resp. (2.2)]. Then either

$$
f(x+y, t+s)-f(x, t) g(y, s)-g(x, t) f(y, s)=0
$$

for all $x, y \in G$ and $t, s>0$, or else there exist $\lambda_{1}, \lambda_{2} \in \mathbb{C}$, not both zero, such that $\lambda_{1} f(x, t)-\lambda_{2} g(x, t)$ satisfies (2.3) [resp. (2.4)]. 
Proof. Assume that $\lambda_{1} f(x, t)-\lambda_{2} g(x, t)$ satisfies (2.3) [resp. (2.4)] only when $\lambda_{1}=$ $\lambda_{2}=0$. Now, it suffices to prove that $f, g$ satisfy (2.19). Let

$$
F(x, y, t, s)=f(x+y, t+s)-f(x, t) g(y, s)-g(x, t) f(y, s) .
$$

Choosing $y_{1}$ and $s_{1}$ with $f\left(y_{1}, s_{1}\right) \neq 0$ we have

$$
g(x, t)=k_{1} f(x, t)+k_{2} f\left(x+y_{1}, t+s_{1}\right)-k_{2} F\left(x, y_{1}, t, s_{1}\right),
$$

where $k_{1}=-\frac{g\left(y_{1}, s_{1}\right)}{f\left(y_{1}, s_{1}\right)}$ and $k_{2}=\frac{1}{f\left(y_{1}, s_{1}\right)}$. From (2.20) and (2.21) we find that

$$
f((x+y)+z,(t+s)+r))
$$

$=f(x+y, t+s) g(z, r)+g(x+y, t+s) f(z, r)+F(x+y, z, t+s, r)$

$=f(x+y, t+s) g(z, r)$

$$
\begin{aligned}
& +\left(k_{1} f(x+y, t+s)+k_{2} f\left(x+y+y_{1}, t+s+s_{1}\right)-k_{2} F\left(x+y, y_{1}, t+s, s_{1}\right)\right) f(z, r) \\
& +F(x+y, z, t+s, r) \\
= & (f(x, t) g(y, s)+g(x, t) f(y, s)+F(x, y, t, s)) g(z, r) \\
& +k_{1}(f(x, t) g(y, s)+g(x, t) f(y, s)+F(x, y, t, s)) f(z, r) \\
& +k_{2}\left(f(x, t) g\left(y+y_{1}, s+s_{1}\right)+g(x, t) f\left(y+y_{1}, s+s_{1}\right)\right. \\
& \left.+F\left(x, y+y_{1}, t, s+s_{1}\right)-F\left(x+y, y_{1}, t+s, s_{1}\right)\right) f(z, r) \\
& +F(x+y, z, t+s, r),
\end{aligned}
$$

and also

$$
\begin{aligned}
& f(x+(y+z), t+(s+r)) \\
& \quad=f(x, t) g(y+z, s+r)+g(x, t) f(y+z, s+r)+F(x, y+z, t, s+r) .
\end{aligned}
$$

From (2.22) and (2.23) we deduce that

$$
\begin{aligned}
& f(x, t)\left(g(y, s) g(z, r)+k_{1} g(y, s) f(z, r)+k_{2} g\left(y+y_{1}, s+s_{1}\right) f(z, r)-g(y+z, s+r)\right) \\
& +g(x, t)\left(f(y, s) g(z, r)+k_{1} f(y, s) f(z, r)+k_{2} f\left(y+y_{1}, s+s_{1}\right) f(z, r)-f(y+z, s+r)\right) \\
& =F(x, y+z, t, s+r)-F(x+y, z, t+s, r)-F(x, y, t, s) g(z, r)-k_{1} F(x, y, t, s) f(z, r) \\
& \quad-k_{2}\left(F\left(x, y+y_{1}, t, s+s_{1}\right)-F\left(x+y, y_{1}, t+s, s_{1}\right)\right) f(z, r) .
\end{aligned}
$$

Fixing $y, z, s, r$ in (2.24), and using (2.1) and (2.20), we find that

$$
\begin{aligned}
& \mid F(x, y+z, t, s+r)-F(x+y, z, t+s, r)-F(x, y, t, s) g(z, r)-k_{1} F(x, y, t, s) f(z, r) \\
& -k_{2}\left(F\left(x, y+y_{1}, t, s+s_{1}\right)-F\left(x+y, y_{1}, t+s, s_{1}\right)\right) f(z, r) \mid \\
& \leq 2 C\left(\frac{1}{t}+\frac{1}{r}\right)^{N}+2 d+C_{1}\left(\frac{1}{t}+\frac{1}{s}\right)^{N}+d_{1}+C_{2}\left(\frac{1}{t}+\frac{1}{s_{1}}\right)^{N}+d_{2} \leq C^{\prime} t^{-N}+d^{\prime}, \\
& \text { where } C^{\prime}=2^{N}\left(2 C+C_{1}+C_{2}\right), d^{\prime}=2^{N}\left(2 C r^{-N}+C_{1} s^{-N}+C_{2} s_{1}^{-N}\right)+2 d+d_{1}+d_{2} \text {. }
\end{aligned}
$$


Similarly, using (2.2) we find that for every $\epsilon>0$ there exists $C_{\epsilon}>0$ such that

$$
\begin{aligned}
\mid F(x, y+z, t, s+r)- & F(x+y, z, t+s, r)-F(x, y, t, s) g(z, r)-k_{1} F(x, y, t, s) f(z, r) \\
& -k_{2}\left(F\left(x, y+y_{1}, t, s+s_{1}\right)-F\left(x+y, y_{1}, t+s, s_{1}\right)\right) f(z, r) \mid \\
\leq & 2 C_{\epsilon} e^{\epsilon(1 / t+1 / r)}+C_{1} C_{\epsilon} e^{\epsilon(1 / t+1 / s)}+C_{2} C_{\epsilon} e^{\epsilon\left(1 / t+1 / s_{1}\right)} \leq C_{\epsilon}^{\prime} e^{\epsilon / t}
\end{aligned}
$$

where $C_{\epsilon}^{\prime}=C_{\epsilon}\left(2 e^{\epsilon / r}+C_{1} e^{\epsilon / s}+C_{2} e^{\epsilon / s_{1}}\right)$.

Thus, by the assumption that $\lambda_{1} f(x, t)-\lambda_{2} g(x, t)$ satisfies (2.3) [resp. (2.4)] only when $\lambda_{1}=\lambda_{2}=0$ we have

$$
\begin{aligned}
& g(y, s) g(z, r)+k_{1} g(y, s) f(z, r)+k_{2} g\left(y+y_{1}, s+s_{1}\right) f(z, r)-g(y+z, s+r) \\
& =f(y, s) g(z, r)+k_{1} f(y, s) f(z, r)+k_{2} f\left(y+y_{1}, s+s_{1}\right) f(z, r)-f(y+z, s+r)=0 .
\end{aligned}
$$

Hence

$$
\begin{aligned}
& F(x, y+z, t, s+r)-F(x+y, z, t+s, r) \\
= & \left(k_{1} F(x, y, t, s)+k_{2} F\left(x, y+y_{1}, t, s+s_{1}\right)-k_{2} F\left(x+y, y_{1}, t+s, s_{1}\right)\right) f(z, r) \\
+ & F(x, y, t, s) g(z, r) .
\end{aligned}
$$

Now, if we fix $x, y, t, s$, the left hand side of (2.25) satisfies (2.3) [resp. (2.4)] as a function of $z$ and $r$. From the right hand side of (2.25), using the assumption that $\lambda_{1} f(x, t)-\lambda_{2} g(x, t)$ satisfies (2.3) [resp. (2.4)] only when $\lambda_{1}=\lambda_{2}=0$ it follows that $F \equiv 0$.

Theorem 2.6. Let $f, g: G \times(0, \infty) \rightarrow \mathbb{C}$ satisfy (2.1) [resp. (2.2)]. Then $(f, g)$ satisfies one of the following:

(i) $f=0$ and $g$ is arbitrary,

(ii) both $f$ and $g$ satisfy (2.3) [resp. (2.4)],

(iii) $f(x, t)=\frac{1}{2 \lambda}(m(x, t)-R(x, t))$ and $g(x, t)=\frac{1}{2}(m(x, t)+R(x, t))$, where $\lambda \in \mathbb{C}$, $m$ is a nonzero exponential function and $R$ is a function satisfying (2.3) [resp. $(2.4)]$.

(iv) we have

$$
\begin{aligned}
& g(x, t)=m_{1}(x) m_{2}(t), \\
& f(x, t)=l(x) m_{1}(x) m_{2}(t)+2 f(0, t / 2) g(0, t / 2) m_{1}(x)+R(x, t),
\end{aligned}
$$

where $m_{1}$ and $m_{2}$ are exponential functions on $G$ and $(0, \infty)$ respectively such that $\left|m_{1}(x)\right|=1$ for all $x \in G$ and $0<\left|m_{2}(t)\right| \leq 1$ for all $t>0, l(x)$ is an additive function on $G$, and $R$ is a function satisfying (2.3) [resp. (2.4)],

(v) $g(x, t)=m(x, t)$ and $f(x, t)=A(x, t) m(x, t)$, where $m$ is a nonzero exponential function and $A$ is a nonzero additive function, 
(vi) $f(x, t)=\frac{1}{2 \lambda}\left(m^{*}(x, t)-m^{* *}(x, t)\right)$ and $g(x, t)=\frac{1}{2}\left(m^{*}(x, t)+m^{* *}(x, t)\right)$, where $\lambda \in \mathbb{C}$, and $m^{*}$ and $m^{* *}$ are nonzero exponential functions.

Proof. In view of Lemma 2.5, we first assume that the equation (2.19) holds. Then Lemma 2.1 shows that the solutions of the sine functional equation (2.19) satisfy one of the cases (i), (iii) with $R \equiv 0$, (v) or (vi). It remains to consider the case when there exist $\lambda_{1}, \lambda_{2} \in \mathbb{C}$, not both zero, such that $\lambda_{1} f(x, t)-\lambda_{2} g(x, t)$ satisfies (2.3) [resp. (2.4)].

First, suppose that $f(\neq 0)$ satisfies (2.3) [resp. (2.4)]. Choosing $y_{0} \in G$ and $s_{0}>0$ such that $f\left(y_{0}, s_{0}\right) \neq 0$, dividing both sides of $(2.1)$ by $\left|f\left(y_{0}, s_{0}\right)\right|$ and using the triangle inequality we have

$$
\begin{aligned}
|g(x, t)| & \leq \frac{1}{\left|f\left(y_{0}, s_{0}\right)\right|}\left(\left|f\left(x+y_{0}, t+s_{0}\right)\right|+\left|f(x, t) g\left(y_{0}, s_{0}\right)\right|+C\left(\frac{1}{t}+\frac{1}{s_{0}}\right)^{N}+d\right) \\
& \leq C_{1}\left(t+s_{0}\right)^{-N}+d_{1}+C_{2} t^{-N}+d_{2}+C_{3}\left(\frac{1}{t}+\frac{1}{s_{0}}\right)^{N}+d_{3} \leq C^{\prime} t^{-N}+d^{\prime}
\end{aligned}
$$

for all $x \in G$ and $t>0$ and for some positive constants $C_{1}, C_{2}, C_{3}, d_{1}, d_{2}, d_{3}, C^{\prime}$ and $d^{\prime}$. Similarly, if $f, g$ satisfy $(2.2)$ we can show that for every $\epsilon>0$ there exists $C_{\epsilon}^{\prime}>0$ such that

$$
|g(x, t)| \leq C_{\epsilon}^{\prime} e^{\epsilon / t}
$$

for all $x \in G$ and $t>0$. Thus, we obtain case (ii).

Secondly, suppose that neither $f$ nor $g$ satisfies (2.3) [resp. (2.4)]. In this case we must have $\lambda_{1} \neq 0$ and $\lambda_{2} \neq 0$. Thus, we can write

$$
g(x, t)=\lambda f(x, t)+R(x, t)
$$

for some $\lambda(\neq 0) \in \mathbb{C}$ and a function $R$ satisfying (2.3) [resp. (2.4)]. Putting (2.26) in (2.1), using the triangle inequality and fixing $y$ and $s$ we have

$$
\begin{aligned}
|f(x+y, t+s)-f(x, t)(R(y, s)+2 \lambda f(y, s))| & \leq|f(y, s) R(x, t)|+C\left(\frac{1}{t}+\frac{1}{s}\right)^{N}+d \\
& \leq C^{\prime} t^{-N}+d^{\prime}\left[\text { resp. } C_{\epsilon}^{\prime} e^{\epsilon / t}\right]
\end{aligned}
$$

for all $x \in G$ and $t>0$ and for some positive constants $C^{\prime}$ and $d^{\prime}$ [resp. for every $\epsilon>0$ there exists a positive constant $C_{\epsilon}^{\prime}$ ]. Applying Lemma 2.2, we obtain

$$
R(y, s)+2 \lambda f(y, s)=m(y, s)
$$

for all $y \in G$ and $s>0$, where $m$ is an exponential function on $G \times(0, \infty)$. Thus, case (iii) follows immediately from (2.26) and (2.27). 
Finally, suppose that $f$ does not satisfy (2.3) [resp. (2.4)] and $g$ satisfies (2.3) [resp. (2.4)]. Then we must have $g \neq 0$. In view of (2.1) [resp. (2.2)], for each $y \in G$ and $s>0, f(x+y, t+s)-f(x, t) g(y, s)$ satisfies (2.3) [resp. (2.4)]. Thus, by Lemma $2.2, g$ is an exponential function. Using Lemma 2.4 we get case (iv).

Corollary 2.7. Let $f, g: \mathbb{R}^{n} \times(0, \infty) \rightarrow \mathbb{C}$ be continuous functions satisfying (2.1) [resp. (2.2)]. Then $f, g$ satisfy one of the following:

(i) $f=0$ and $g$ is arbitrary,

(ii) both $f$ and $g$ satisfy (2.3) [resp. (2.4)],

(iii) $f(x, t)=\frac{1}{2 \lambda}\left(e^{c \cdot x+b t}-R(x, t)\right)$ and $g(x, t)=\frac{1}{2}\left(e^{c \cdot x+b t}+R(x, t)\right)$, where $\lambda, b \in \mathbb{C}$, $c \in \mathbb{C}^{n}$ and $R$ is a function satisfying (2.3) [resp. (2.4)],

(iv) $g(x, t)=e^{i c \cdot x+b t}$ and $f(x, t)=a \cdot x e^{i c \cdot x+b t}+2 f(0, t / 2) e^{i c \cdot x+\frac{1}{2} b t}+R(x, t)$, where $c \in \mathbb{R}^{n}, a \in \mathbb{C}^{n}, b \in \mathbb{C}$, and $R$ is a function satisfying (2.3) [resp. (2.4)],

(v) $g(x, t)=e^{c \cdot x+b t}$ and $f(x, t)=(a \cdot x+d t) e^{c \cdot x+b t}$, where $a, c \in \mathbb{C}^{n}$ and $b, d \in \mathbb{C}$,

(vi) $f(x, t)=\frac{1}{2 \lambda}\left(e^{c_{1} \cdot x+b_{1} t}-e^{c_{2} \cdot x+b_{2} t}\right)$ and $g(x, t)=\frac{1}{2}\left(e^{c_{1} \cdot x+b_{1} t}-e^{c_{2} \cdot x+b_{2} t}\right)$, where $\lambda, b_{1}, b_{2} \in \mathbb{C}$ and $c_{1}, c_{2} \in \mathbb{C}^{n}$.

Proof. It follows from the continuity of $f$ and $g$ that the exponential functions $m, m^{*}, m^{* *}$ and the additive function $A$ in (iii)-(vi) of Theorem 2.6 are continuous. Also, in view of the proof of Lemma 2.4, the additive function $l(x)$ in (iv) of Theorem 2.6 is continuous since it is the uniform limit of a sequence of continuous functions. Now, it is well known that the continuous solutions $A: \mathbb{R}^{n} \times(0, \infty) \rightarrow \mathbb{C}$ of the Cauchy functional equation

$$
A(x+y, t+s)=A(x, t)+A(y, s)
$$

are of the form $A(x, t)=c \cdot x+b t$, and the continuous solutions $m: \mathbb{R}^{n} \times(0, \infty) \rightarrow \mathbb{C}$ of the exponential functional equation

$$
m(x+y, t+s)=m(x, t) m(y, s)
$$

are of the form $m(x, t)=e^{c \cdot x+b t}$ for some $c \in \mathbb{C}^{n}, b \in \mathbb{C}$. If, in particular, $m$ satisfies (2.3) [resp. (2.4)], then $c=i a$ for some $a \in \mathbb{R}^{n}$ and $\Re b<0$. Thus, cases (i)-(vi) follow immediately from (i)-(vi) of Theorem 2.6, respectively.

\section{$\S 3$. Bounded distributions and hyperfunctions}

We first introduce the spaces $\mathcal{S}^{\prime}$ of Schwartz tempered distributions and $\mathcal{G}^{\prime}$ of Gelfand hyperfunctions (see [20-22,26,33] for more details). We use the notations $|\alpha|=\alpha_{1}+\cdots+\alpha_{n}, \alpha !=\alpha_{1} ! \cdots \alpha_{n} !,|x|=\sqrt{x_{1}^{2}+\cdots+x_{n}^{2}}, x^{\alpha}=x_{1}^{\alpha_{1}} \cdots x_{n}^{\alpha_{n}}$ and 
$\partial^{\alpha}=\partial_{1}^{\alpha_{1}} \ldots \partial_{n}^{\alpha_{n}}$, for $x=\left(x_{1}, \ldots, x_{n}\right) \in \mathbb{R}^{n}, \alpha=\left(\alpha_{1}, \ldots, \alpha_{n}\right) \in \mathbb{N}_{0}^{n}$, where $\mathbb{N}_{0}$ is the set of nonnegative integers and $\partial_{j}=\partial / \partial x_{j}$.

Definition 3.1 ([33]). We denote by $\mathcal{S}$ or $\mathcal{S}\left(\mathbb{R}^{n}\right)$ the Schwartz space of all infinitely differentiable functions $\varphi$ on $\mathbb{R}^{n}$ such that

$$
\|\varphi\|_{\alpha, \beta}=\sup _{x}\left|x^{\alpha} \partial^{\beta} \varphi(x)\right|<\infty
$$

for all $\alpha, \beta \in \mathbb{N}_{0}^{n}$, equipped with the topology defined by the seminorms $\|\cdot\|_{\alpha, \beta}$. The elements of $\mathcal{S}$ are called rapidly decreasing functions and the elements of the dual space $\mathcal{S}^{\prime}$ are tempered distributions.

Definition $3.2([20,21])$. We denote by $\mathcal{G}$ or $\mathcal{G}\left(\mathbb{R}^{n}\right)$ the Gelfand space of all infinitely differentiable functions $\varphi$ in $\mathbb{R}^{n}$ such that

$$
\|\varphi\|_{h, k}=\sup _{x \in \mathbb{R}^{n}, \alpha, \beta \in \mathbb{N}_{0}^{n}} \frac{\left|x^{\alpha} \partial^{\beta} \varphi(x)\right|}{h^{|\alpha|} k^{|\beta|} \alpha !^{1 / 2} \beta !^{1 / 2}}<\infty
$$

for some $h, k>0$. We say that $\varphi_{j} \rightarrow 0$ as $j \rightarrow \infty$ if $\left\|\varphi_{j}\right\|_{h, k} \rightarrow 0$ as $j \rightarrow \infty$ for some $h, k$; we denote by $\mathcal{G}^{\prime}$ the strong dual space of $\mathcal{G}$ and call its elements Gelfand hyperfunctions.

As a generalization of the space $L^{\infty}$ of bounded measurable functions, L. Schwartz introduced the space $\mathcal{D}_{L^{\infty}}^{\prime}$ of bounded distributions as a subspace of tempered distributions.

Definition 3.3 ([33]). We denote by $\mathcal{D}_{L^{1}}\left(\mathbb{R}^{n}\right)$ the space of smooth functions on $\mathbb{R}^{n}$ such that $\partial^{\alpha} \varphi \in L^{1}\left(\mathbb{R}^{n}\right)$ for all $\alpha \in \mathbb{N}_{0}^{n}$, equipped with the topology defined by the countable family of seminorms

$$
\|\varphi\|_{m}=\sum_{|\alpha| \leq m}\left\|\partial^{\alpha} \varphi\right\|_{L^{1}}, \quad m \in \mathbb{N}_{0} .
$$

We denote by $\mathcal{D}_{L^{\infty}}^{\prime}$ the strong dual space of $\mathcal{D}_{L^{1}}$ and call its elements bounded distributions.

Generalizing bounded distributions, the space $\mathcal{A}_{L^{\infty}}^{\prime}$ of bounded hyperfunctions has been introduced as a subspace of $\mathcal{G}^{\prime}$.

Definition 3.4 ([16]). We denote by $\mathcal{A}_{L^{1}}$ the space of smooth functions $\varphi$ on $\mathbb{R}^{n}$ satisfying

$$
\|\varphi\|_{h}=\sup _{\alpha} \frac{\left\|\partial^{\alpha} \varphi\right\|_{L^{1}}}{h^{|\alpha|} \alpha !}<\infty
$$


for some constant $h>0$. We say that $\varphi_{j} \rightarrow 0$ in $\mathcal{A}_{L^{1}}$ as $j \rightarrow \infty$ if there is a positive constant $h$ such that

$$
\left\|\varphi_{j}\right\|_{h} \rightarrow 0 \quad \text { as } j \rightarrow \infty
$$

We denote by $\mathcal{A}_{L^{\infty}}^{\prime}$ the strong dual space of $\mathcal{A}_{L^{1}}$.

It is well known that the following topological inclusions hold:

$$
\begin{array}{ll}
\mathcal{G} \hookrightarrow \mathcal{S} \hookrightarrow \mathcal{D}_{L^{1}}, & \mathcal{D}_{L^{\infty}}^{\prime} \hookrightarrow \mathcal{S}^{\prime} \hookrightarrow \mathcal{G}^{\prime}, \\
\mathcal{G} \hookrightarrow \mathcal{A}_{L^{1}} \hookrightarrow \mathcal{D}_{L^{1}}, & \mathcal{D}_{L^{\infty}}^{\prime} \hookrightarrow \mathcal{A}_{L^{\infty}}^{\prime} \hookrightarrow \mathcal{G}^{\prime} .
\end{array}
$$

It is known that the space $\mathcal{G}\left(\mathbb{R}^{n}\right)$ consists of all infinitely differentiable functions $\varphi$ on $\mathbb{R}^{n}$ which can be extended to an entire function on $\mathbb{C}^{n}$ satisfying

$$
|\varphi(x+i y)| \leq C \exp \left(-a|x|^{2}+b|y|^{2}\right), \quad x, y \in \mathbb{R}^{n},
$$

for some $a, b, C>0$ (see [20]).

Definition 3.5. Let $u_{j} \in \mathcal{G}^{\prime}\left(\mathbb{R}^{n_{j}}\right)$ for $j=1,2$. Then the tensor product $u_{1} \otimes u_{2}$ of $u_{1}$ and $u_{2}$, defined by

$$
\left\langle u_{1} \otimes u_{2}, \varphi\left(x_{1}, x_{2}\right)\right\rangle=\left\langle u_{1},\left\langle u_{2}, \varphi\left(x_{1}, x_{2}\right)\right\rangle\right\rangle
$$

for $\varphi\left(x_{1}, x_{2}\right) \in \mathcal{G}\left(\mathbb{R}^{n_{1}} \times \mathbb{R}^{n_{2}}\right)$, belongs to $\mathcal{G}^{\prime}\left(\mathbb{R}^{n_{1}} \times \mathbb{R}^{n_{2}}\right)$.

\section{$\S 4$. Stability in distributions and hyperfunctions}

In this section we consider the Hyers-Ulam stability for the sine functional equation in the space of distributions and hyperfunctions,

$$
u \circ S-u \otimes v-v \otimes u \in \mathcal{D}_{L^{\infty}}^{\prime}\left(\mathbb{R}^{2 n}\right)\left[\operatorname{resp} . \mathcal{A}_{L^{\infty}}^{\prime}\left(\mathbb{R}^{2 n}\right)\right],
$$

where $\otimes$ denotes the tensor product of generalized functions, $S(x, y)=x+y$ for $x, y \in \mathbb{R}^{n}$, and the pullback $u \circ S$ is defined by

$$
\langle u \circ S, \varphi(x, y)\rangle=\left\langle u, \int \varphi(x-y, y) d y\right\rangle, \quad \varphi \in \mathcal{G}\left(\mathbb{R}^{2 n}\right) .
$$

In view of Definition 3.2, it is easy to see that if $\varphi_{j}(x, y) \in \mathcal{G}\left(\mathbb{R}^{2 n}\right)$ is a sequence such that $\varphi_{j} \rightarrow 0$ in $\mathcal{G}\left(\mathbb{R}^{n}\right)$ as $j \rightarrow \infty$, then $\int \varphi_{j}(x-y, y) d y \rightarrow 0$ in $\mathcal{G}\left(\mathbb{R}^{n}\right)$ as $j \rightarrow \infty$. Thus, $u \circ S \in \mathcal{G}^{\prime}\left(\mathbb{R}^{2 n}\right)$.

For the proof of our theorems we employ the $n$-dimensional heat kernel $E_{t}(x)$ given by

$$
E_{t}(x)=(4 \pi t)^{-n / 2} \exp \left(-|x|^{2} / 4 t\right), \quad t>0
$$


In view of $(3.2)$, we can see that the heat kernel $E_{t}$ belongs to the Gelfand space $\mathcal{G}\left(\mathbb{R}^{n}\right)$ for each $t>0$. Thus, for each $u \in \mathcal{G}^{\prime}\left(\mathbb{R}^{n}\right)$, the convolution $\left(u * E_{t}\right)(x):=$ $\left\langle u_{y}, E_{t}(x-y)\right\rangle$ is well defined. We call $\left(u * E_{t}\right)(x)$ the Gauss transform of $u$. From now on we denote by $\tilde{u}(x, t)$ the Gauss transform of $u$. It is well known that $\tilde{u}(x, t)$ is a smooth solution of the heat equation such that $\tilde{u}(\cdot, t) \rightarrow u$ in the weak* topology as $t \rightarrow 0^{+}$, i.e.,

$$
\langle u, \varphi\rangle=\lim _{t \rightarrow 0^{+}} \int \tilde{u}(x, t) \varphi(x) d x
$$

for all $\varphi \in \mathcal{G}$.

Example. Let $u(x)=x^{\alpha}, \alpha \in \mathbb{N}_{0}^{n}, v(x)=e^{c \cdot x}, w(x)=a \cdot x e^{c \cdot x}, a=\left(a_{1}, \ldots, a_{n}\right)$, $c=\left(c_{1}, \ldots, c_{n}\right) \in \mathbb{C}^{n}$. Then $u, v, w \in \mathcal{G}^{\prime}\left(\mathbb{R}^{n}\right)$ and simple calculations show that

$$
\begin{aligned}
\tilde{u}(x, t) & =\left[\xi^{\alpha} * E_{t}(\xi)\right](x)=\alpha ! \sum_{0 \leq 2 \gamma \leq \alpha} \frac{t^{|\gamma|} x^{\alpha-2 \gamma}}{\gamma !(\alpha-2 \gamma) !}, \\
\tilde{v}(x, t) & =\left[e^{c \cdot \xi} * E_{t}(\xi)\right](x)=e^{c \cdot x+\left(c_{1}^{2}+\cdots+c_{n}^{2}\right) t}, \\
\tilde{w}(x, t) & =\left[a \cdot \xi e^{c \cdot \xi} * E_{t}(\xi)\right](x)=(a \cdot x+2 a \cdot c t) e^{c \cdot x+\left(c_{1}^{2}+\cdots+c_{n}^{2}\right) t} .
\end{aligned}
$$

The proof of Theorem 2.3 of [15] works even when $p=\infty$, i.e., we obtain the following.

Lemma 4.1 ([15]). The Gauss transform $\tilde{u}(x, t):=(u * E)(x, t)$ of $u \in \mathcal{D}_{L^{\infty}}^{\prime}\left(\mathbb{R}^{n}\right)$ is a smooth solution of the heat equation $\left(\Delta-\partial / \partial_{t}\right) \tilde{u}=0$ satisfying:

(i) There exist constants $C>0$ and $N \geq 0$ such that

$$
|\tilde{u}(x, t)| \leq C t^{-N} \quad \text { for all } x \in \mathbb{R}^{n} \text { and } t>0 .
$$

(ii) $\tilde{u}(\cdot, t) \rightarrow u$ as $t \rightarrow 0^{+}$in the sense that for every $\varphi \in \mathcal{D}_{L^{1}}$,

$$
\langle u, \varphi\rangle=\lim _{t \rightarrow 0^{+}} \int \tilde{u}(x, t) \varphi(x) d x .
$$

Conversely, every smooth solution $\tilde{u}(x, t)$ of the heat equation satisfying the estimate (4.2) can be uniquely expressed as $\tilde{u}(x, t)=(u * E)(x, t)$ for some $u \in \mathcal{D}_{L^{\infty}}^{\prime}\left(\mathbb{R}^{n}\right)$

The following lemma is a special case of Theorem 3.5 of [16] when $p=\infty$ (in [16], the space $\mathcal{A}_{L^{\infty}}^{\prime}\left(\mathbb{R}^{n}\right)$ is denoted by $\mathcal{B}_{L^{\infty}}\left(\mathbb{R}^{n}\right)$ ).

Lemma $4.2([16])$. The Gauss transform $\tilde{u}(x, t):=(u * E)(x, t)$ of $u \in \mathcal{A}_{L^{\infty}}^{\prime}\left(\mathbb{R}^{n}\right)$ is a smooth solution of the heat equation $\left(\Delta-\partial / \partial_{t}\right) \tilde{u}=0$ satisfying: 
(i) For every $\epsilon>0$ there exists a constant $C_{\epsilon}>0$ such that

$$
|\tilde{u}(x, t)| \leq C_{\epsilon} e^{\epsilon / t} \quad \text { for all } x \in \mathbb{R}^{n}, t>0 .
$$

(ii) $\tilde{u}(\cdot, t) \rightarrow u$ as $t \rightarrow 0^{+}$in the sense that for every $\varphi \in \mathcal{A}_{L^{1}}$,

$$
\langle u, \varphi\rangle=\lim _{t \rightarrow 0^{+}} \int \tilde{u}(x, t) \varphi(x) d x .
$$

Conversely, every smooth solution $\tilde{u}$ of the heat equation satisfying (4.3) can be uniquely expressed as $\tilde{u}(x, t)=(u * E)(x, t)$ for some $u \in \mathcal{A}_{L^{\infty}}^{\prime}\left(\mathbb{R}^{n}\right)$.

The following structure theorem for bounded distributions is well known. We refer the reader to [33, Theorem 25 in Chapter 6].

Lemma 4.3. Every $u \in \mathcal{D}_{L^{\infty}}^{\prime}\left(\mathbb{R}^{n}\right)$ can be expressed as

$$
u=\sum_{|\alpha| \leq p} \partial^{\alpha} f_{\alpha}
$$

for some $p \in \mathbb{N}_{0}$ where $f_{\alpha} \in L^{\infty}\left(\mathbb{R}^{n}\right)$ for all $|\alpha| \leq p$. The equality (4.4) implies that

$$
\langle u, \varphi\rangle=\sum_{|\alpha| \leq p}(-1)^{|\alpha|} \int f_{\alpha}(x) \partial^{\alpha} \varphi(x) d x
$$

for all $\varphi \in \mathcal{D}_{L^{1}}$

As a special case of Theorem 3.4 of [16] when $p=\infty$ we obtain the following.

Lemma 4.4 ([16]). Every $u \in \mathcal{A}_{L^{\infty}}^{\prime}\left(\mathbb{R}^{n}\right)$ can be expressed as

$$
u=\left(\sum_{k=0}^{\infty} a_{k} \Delta^{k}\right) g+h
$$

where $\Delta$ denotes the Laplacian, $g, h$ are bounded continuous functions on $\mathbb{R}^{n}$ and $a_{k}, k=0,1,2, \ldots$, satisfy the following estimates: for every $L>0$ there exists $C>0$ such that

$$
\left|a_{k}\right| \leq C L^{k} / k !^{2}
$$

for all $k=0,1,2, \ldots$

The following properties of the heat kernel will be useful; they can be found in [26]. Here we give a slightly modified proof.

Proposition $4.5([26])$. For each $t>0, E_{t}(\cdot)$ is an entire function and the following estimate holds: there exists $C>0$ such that for all $x \in \mathbb{R}^{n}$ and $t>0$,

$$
\left|\partial_{x}^{\alpha} E_{t}(x)\right| \leq C^{|\alpha|} t^{-(n+|\alpha|) / 2} \alpha !^{1 / 2} \exp \left(-|x|^{2} / 8 t\right) .
$$


Also for all $x \in \mathbb{R}^{n}$ and $t, s>0$,

$$
\left(E_{t} * E_{s}\right)(x):=\int E_{t}(x-y) E_{s}(y) d y=E_{t+s}(x) .
$$

Proof. The equality (4.7) is proved by the well-known calculation which we omit. We prove (4.6) for $n=1$. By the Cauchy integral formula we have

$$
\frac{d^{k}}{d x^{k}} E_{t}(x)=\frac{k !}{2 \pi i} \int_{C_{r}} \frac{E_{t}(z)}{(z-x)^{k+1}} d z
$$

where $C_{r}$ is the circle of radius $r$ with center at $z=x$. Using (4.8) and the triangle inequality we obtain

$$
\begin{aligned}
\left|\partial^{k} E_{t}(x)\right| & \leq \frac{k !}{\sqrt{4 \pi t} r^{k}} \sup _{z \in C_{r}}\left|\exp \left(-z^{2} / 4 t\right)\right| \\
& \leq \frac{k !}{\sqrt{4 \pi t} r^{k}} \sup _{0 \leq \theta \leq 2 \pi} \exp \left(\frac{-(x+r \cos \theta)^{2}+r^{2} \sin ^{2} \theta}{4 t}\right) \\
& \leq \frac{k !}{\sqrt{4 \pi t} r^{k}} \exp \left(\frac{-\frac{1}{2} x^{2}+r^{2}}{4 t}\right)=\frac{k !}{\sqrt{4 \pi t} r^{k}} \exp \left(\frac{r^{2}}{4 t}\right) \exp \left(-\frac{x^{2}}{8 t}\right) .
\end{aligned}
$$

The right hand side of (4.9) attains its minimum at $r=\sqrt{2 k t}$. Thus, (4.9) reduces to

$$
\left|\partial^{k} E_{t}(x)\right| \leq \frac{(e / 2)^{k / 2}}{\sqrt{4 \pi}} k !^{1 / 2} t^{-(1+k) / 2} \exp \left(-\frac{x^{2}}{8 t}\right) .
$$

The general case is proved in the same manner.

Now, we state and prove the main theorems.

Theorem 4.6. Let $u, v \in \mathcal{G}^{\prime}\left(\mathbb{R}^{n}\right)$. Then $(u, v)$ satisfies (4.1) if and only if $(u, v)$ satisfies one of the following:

(i) $u=0$ and $v$ is arbitrary,

(ii) $u$ and $v$ are bounded distributions [resp. bounded hyperfunctions],

(iii) $u=\frac{1}{2 \lambda}\left(e^{c \cdot x}-w_{0}\right)$ and $v=\frac{1}{2}\left(e^{c \cdot x}+w_{0}\right)$ for some $c \in \mathbb{C}^{n}, \lambda \in \mathbb{C}$ and $w_{0} \in \mathcal{D}_{L^{\infty}}^{\prime}\left(\mathbb{R}^{n}\right)\left[\right.$ resp. $\left.\mathcal{A}_{L^{\infty}}^{\prime}\left(\mathbb{R}^{n}\right)\right]$,

(iv) $u=a \cdot x e^{i c \cdot x}+w_{0}$ and $v=e^{i c \cdot x}$ for some $a \in \mathbb{C}^{n}, c \in \mathbb{R}^{n}$ and $w_{0} \in$ $\mathcal{D}_{L^{\infty}}^{\prime}\left(\mathbb{R}^{n}\right)\left[\operatorname{resp} . \mathcal{A}_{L^{\infty}}^{\prime}\left(\mathbb{R}^{n}\right)\right]$,

(v) $u=a \cdot x e^{c \cdot x}$ and $v=e^{c \cdot x}$ for some $a, c \in \mathbb{C}^{n}$,

(vi) $u=\frac{1}{2 \lambda}\left(e^{c_{1} \cdot x}-e^{c_{2} \cdot x}\right)$ and $v=\frac{1}{2}\left(e^{c_{1} \cdot x}+e^{c_{2} \cdot x}\right)$ for some $c_{1}, c_{2} \in \mathbb{C}^{n}$ and $\lambda \in \mathbb{C}$. 
Proof. We first obtain the following inequality from (4.1): there exist $C, d>0$ [resp. for every $\epsilon>0$ there exists $C_{\epsilon}>0$ ] such that

$$
\begin{aligned}
\mid \tilde{u}(x+y, t+s)-\tilde{u}(x, t) \tilde{v} & (y, s)-\tilde{v}(x, t) \tilde{u}(y, s) \mid \\
& \leq C\left(\frac{1}{t}+\frac{1}{s}\right)^{N}+d\left[\operatorname{resp} . C_{\epsilon} e^{\epsilon(1 / t+1 / s)}\right]
\end{aligned}
$$

where $\tilde{u}, \tilde{v}$ are the Gauss transforms of $u, v$, respectively, given in Lemma 4.1.

Convolving with the tensor product $E_{t}(x) E_{s}(y)$ of the $n$-dimensional heat kernels on the left hand side of (4.1), in view of the semigroup property $\left(E_{t} * E_{s}\right)(x)$ $=E_{t+s}(x)$ of the heat kernel we have

$$
\begin{array}{r}
{\left[(u \circ S) *\left(E_{t}(\xi) E_{s}(\eta)\right)\right](x, y)=\left\langle u_{\xi}, \int E_{t}(x-\xi+\eta) E_{s}(y-\eta) d \eta\right\rangle} \\
=\left\langle u_{\xi},\left(E_{t} * E_{s}\right)(x+y-\xi)\right\rangle=\tilde{u}(x+y, t+s) .
\end{array}
$$

Similarly,

$$
\begin{aligned}
& {\left[(u \otimes v) *\left(E_{t}(\xi) E_{s}(\eta)\right)\right](x, y)=\tilde{u}(x, t) \tilde{v}(y, s),} \\
& {\left[(v \otimes u) *\left(E_{t}(\xi) E_{s}(\eta)\right)\right](x, y)=\tilde{v}(x, t) \tilde{u}(y, s),}
\end{aligned}
$$

where $\tilde{u}(x, t), \tilde{v}(x, t)$ are the Gauss transforms of $u, v$, respectively.

Let $w:=u \circ S-u \otimes v-v \otimes u$. Then $w \in \mathcal{D}_{L^{\infty}}^{\prime}\left(\mathbb{R}^{2 n}\right)\left[\operatorname{resp} . \mathcal{A}_{L^{\infty}}^{\prime}\left(\mathbb{R}^{2 n}\right)\right]$. First, we suppose that $w \in \mathcal{D}_{L^{\infty}}^{\prime}\left(\mathbb{R}^{2 n}\right)$. Using (4.4) and (4.6) we have

$$
\begin{aligned}
& \left|\left[w *\left(E_{t}(\xi) E_{s}(\eta)\right)\right](x, y)\right| \leq \sum_{|\alpha| \leq p}\left|\left[\partial^{\alpha} f_{\alpha} *\left(E_{t}(\xi) E_{s}(\eta)\right)\right](x, y)\right| \\
& \quad \leq \sum_{|\alpha| \leq p}\left|\left[f_{\alpha} * \partial_{\xi, \eta}^{\alpha}\left(E_{t}(\xi) E_{s}(\eta)\right)\right](x, y)\right| \leq \sum_{|\alpha| \leq p}\left\|f_{\alpha}\right\|_{L^{\infty}}\left\|\partial_{\xi, \eta}^{\alpha}\left(E_{t}(\xi) E_{s}(\eta)\right)\right\|_{L^{1}} \\
& \quad \leq C_{1} \sum_{|\beta|+|\gamma| \leq p}\left\|\partial_{\xi}^{\beta} E_{t}(\xi)\right\|_{L^{1}}\left\|\partial_{\eta}^{\gamma} E_{s}(\eta)\right\|_{L^{1}} \\
& \quad \leq C_{2} \sum_{|\beta|+|\gamma| \leq p} t^{-(n+|\beta|) / 2} s^{-(n+|\gamma|) / 2} \leq C\left(\frac{1}{t}+\frac{1}{s}\right)^{N}+d
\end{aligned}
$$

where $N=n+p / 2$ and the constants $C$ and $d$ depend only on $p$.

Secondly, we suppose that $w \in \mathcal{A}_{L^{\infty}}^{\prime}\left(\mathbb{R}^{2 n}\right)$. Then using (4.6) we have

$$
\begin{aligned}
\left\|\Delta^{k}\left(E_{t}(\xi) E_{s}(\eta)\right)\right\|_{L^{1}} & \leq \sum_{|\alpha|=k} \frac{k !}{\alpha !}\left\|\partial^{2 \alpha}\left(E_{t}(\xi) E_{s}(\eta)\right)\right\|_{L^{1}} \\
& \leq \sum_{|\beta|+|\gamma|=k} \frac{k !}{\beta ! \gamma !}\left\|\partial_{\xi}^{2 \beta} E_{t}(\xi)\right\|_{L^{1}}\left\|\partial_{\eta}^{2 \gamma} E_{s}(\eta)\right\|_{L^{1}}
\end{aligned}
$$




$$
\begin{aligned}
& \leq \sum_{|\beta|+|\gamma|=k} \frac{k !(2 \beta) !^{1 / 2}(2 \gamma) !^{1 / 2} M^{2 k}}{\beta ! \gamma !} t^{-n / 2-|\beta|} s^{-n / 2-|\gamma|} \\
& \leq \sum_{|\beta|+|\gamma|=k} k !(2 M)^{2 k} t^{-n / 2-|\beta|} s^{-n / 2-|\gamma|} \leq k !(2 \sqrt{n} M)^{2 k}(1 / t+1 / s)^{n+k} .
\end{aligned}
$$

Now, by the structure (4.5) of bounded hyperfunctions together with the growth condition on $a_{k}, k=0,1,2, \ldots$, we have

$$
\begin{aligned}
\mid\left[w * \left(E_{t}(\xi)\right.\right. & \left.\left.E_{s}(\eta)\right)\right](x, y) \mid \\
& \leq \sum_{k=0}^{\infty}\left\|a_{k}\left(\Delta^{k} g\right) *\left(E_{t}(\xi) E_{s}(\eta)\right)\right\|_{L^{\infty}}+\left\|h *\left(E_{t}(\xi) E_{s}(\eta)\right)\right\|_{L^{\infty}} \\
& \leq\|g\|_{L^{\infty}} \sum_{k=0}^{\infty}\left\|a_{k} \Delta^{k}\left(E_{t}(\xi) E_{s}(\eta)\right)\right\|_{L^{1}}+\|h\|_{L^{\infty}}\left\|E_{t}(\xi) E_{s}(\eta)\right\|_{L^{1}} \\
& \leq C_{1} \sum_{k=0}^{\infty} \frac{1}{k !}\left(4 n M^{2} L\right)^{k}(1 / t+1 / s)^{n+k}+\|h\|_{L^{\infty}} \\
& \leq C_{2} \sum_{k=0}^{\infty} \frac{1}{k !} \epsilon^{k}(1 / t+1 / s)^{n+k}+\|h\|_{L^{\infty}} \leq C_{\epsilon} e^{\epsilon(1 / t+1 / s)}
\end{aligned}
$$

where $L$ is taken so that $4 n M^{2} L<\epsilon$ and the constant $C_{\epsilon}$ depends only on $w$ and $\epsilon$. Thus, we have the inequality (4.10). Replacing $f$ by $\tilde{u}, g$ by $\tilde{v}$ and using Corollary 2.7, we obtain one of the following:

(I) $\tilde{u}=0$ and $\tilde{v}$ is arbitrary,

(II) both $\tilde{u}$ and $\tilde{v}$ satisfy (2.3) [resp. (2.4)],

(III) $\tilde{u}(x, t)=\frac{1}{2 \lambda}\left(e^{c \cdot x+b t}-R(x, t)\right)$ and $\tilde{v}(x, t)=\frac{1}{2}\left(e^{c \cdot x+b t}+R(x, t)\right)$, where $\lambda, b \in \mathbb{C}, c \in \mathbb{C}^{n}$ and $R$ is a function satisfying (2.3) [resp. (2.4)],

(IV) $\tilde{v}(x, t)=e^{i c \cdot x+b t}$ and $\tilde{u}(x, t)=a \cdot x e^{i c \cdot x+b t}+2 \tilde{u}(0, t / 2) e^{i c \cdot x+\frac{1}{2} b t}+R(x, t)$ where $c \in \mathbb{R}^{n}, a \in \mathbb{C}^{n}, d \in \mathbb{C}$, and $R$ is a function satisfying (2.3) [resp. (2.4)],

(V) $\tilde{v}(x, t)=e^{c \cdot x+b t}$ and $\tilde{u}(x, t)=(a \cdot x+d t) e^{c \cdot x+b t}$ where $a, c \in \mathbb{C}^{n}$ and $b, d \in \mathbb{C}$,

(VI) $\tilde{u}(x, t)=\frac{1}{2 \lambda}\left(e^{c_{1} \cdot x+b_{1} t}-e^{c_{2} \cdot x+b_{2} t}\right)$ and $\tilde{v}(x, t)=\frac{1}{2}\left(e^{c_{1} \cdot x+b_{1} t}-e^{c_{2} \cdot x+b_{2} t}\right)$, where $\lambda, b_{1}, b_{2} \in \mathbb{C}$ and $c_{1}, c_{2} \in \mathbb{C}^{n}$.

Case (i) is obvious. By Lemma 4.1, case (II) implies (ii). From (III) we have

$$
\tilde{v}(x, t)-\lambda \tilde{u}(x, t)=R(x, t) .
$$

Thus, $R(x, t)$ is a solution of the heat equation. Letting $t \rightarrow 0^{+}$in (III) and using Lemma 4.1 we have $R(x, t) \rightarrow w_{0}$ in the weak* topology for some $w_{0}$ in 
$\mathcal{D}_{L^{\infty}}^{\prime}\left(\mathbb{R}^{n}\right)\left[\right.$ resp. $\left.\mathcal{A}_{L^{\infty}}^{\prime}\left(\mathbb{R}^{n}\right)\right]$, which gives case (iii). Letting $t \rightarrow 0^{+}$in (V) and (VI) we get (v) and (vi), respectively.

Finally, we prove that (IV) implies (iv). Since $\tilde{v}(x, t)=e^{i c \cdot x+b t}$ in (IV) is a solution of the heat equation, we have $b=-\left(c_{1}^{2}+\cdots+c_{n}^{2}\right):=-|c|^{2}$, where $c=\left(c_{1}, \ldots, c_{n}\right)$. Let

$$
R_{1}(x, t)=\tilde{u}(x, t)-(a \cdot x+2 i a \cdot c t) e^{i c \cdot x-|c|^{2} t} .
$$

Then $R_{1}$ is a solution of the heat equation. Also from (IV) we have

$$
R_{1}(x, t)=-2 i a \cdot c t e^{i c \cdot x-|c|^{2} t}+2 \tilde{u}(0, t / 2) e^{i c \cdot x+\frac{1}{2} b t}+R(x, t) .
$$

By the continuity of $\tilde{u}$, there exists $M>0$ such that $|\tilde{u}(0, t+1)| \leq M$ for all $t \in[0,1]$. Putting $x=y=0, s=1$ in (4.10), dividing the result by $|\tilde{v}(0,1)|$, and using the triangle inequality we obtain

$$
\begin{aligned}
|\tilde{u}(0, t)| & \leq \frac{\left|\tilde{u}(0, t+1)-\tilde{u}(0,1) e^{-|c|^{2} t}\right|+C(1 / t+1)^{N}+d}{|\tilde{v}(0,1)|} \\
& \leq \frac{2 M+C(1 / t+1)^{N}+d}{|\tilde{v}(0,1)|} \leq C^{\prime} t^{-N}\left[\text { resp. } C_{\epsilon} e^{\epsilon / t}\right]
\end{aligned}
$$

for all $t \in(0,1)$. From (4.14)-(4.16) we can see that $R_{1}$ is a solution of the heat equation satisfying (4.2) [resp. (4.3)]. By Lemma 4.1 [resp. Lemma 4.2], there exists $w_{0} \in \mathcal{D}_{L^{\infty}}^{\prime}\left(\mathbb{R}^{n}\right)$ [resp. $\left.\mathcal{A}_{L^{\infty}}^{\prime}\left(\mathbb{R}^{n}\right)\right]$ such that $R_{1} \rightarrow w_{0}$ as $t \rightarrow 0^{+}$. Thus, letting $t \rightarrow 0^{+}$in (IV) and using (4.15) we get (iv).

Now, we consider the stability condition (4.1) in the space of Schwartz tempered distributions. Recall that the following topological inclusions hold:

$$
\begin{array}{ll}
\mathcal{G} \hookrightarrow \mathcal{S} \hookrightarrow \mathcal{D}_{L^{1}}, & \mathcal{D}_{L^{\infty}}^{\prime} \hookrightarrow \mathcal{S}^{\prime} \hookrightarrow \mathcal{G}^{\prime}, \\
\mathcal{G} \hookrightarrow \mathcal{A}_{L^{1}} \hookrightarrow \mathcal{D}_{L^{1}}, & \mathcal{D}_{L^{\infty}}^{\prime} \hookrightarrow \mathcal{A}_{L^{\infty}}^{\prime} \hookrightarrow \mathcal{G}^{\prime} .
\end{array}
$$

In view of these inclusions, if $u, v \in \mathcal{S}^{\prime}\left(\mathbb{R}^{n}\right)$ it is natural to consider the condition

$$
u \circ S-u \otimes v-v \otimes u \in \mathcal{D}_{L^{\infty}}^{\prime}\left(\mathbb{R}^{2 n}\right) .
$$

Theorem 4.7. Let $u, v \in \mathcal{S}^{\prime}\left(\mathbb{R}^{n}\right)$. Then $(u, v)$ satisfies (4.17) if and only if $(u, v)$ satisfies one of the following:

(i) $u=0$ and $v$ is arbitrary,

(ii) $u$ and $v$ are bounded distributions,

(iii) $u=a \cdot x e^{i c \cdot x}+w_{0}$ and $v=e^{i c \cdot x}$ for some $a \in \mathbb{C}^{n}, c \in \mathbb{R}^{n}$ and $w_{0} \in \mathcal{D}_{L^{\infty}}^{\prime}\left(\mathbb{R}^{n}\right)$. 
Proof. It is easy to see that $e^{c \cdot x} \in \mathcal{S}^{\prime}\left(\mathbb{R}^{n}\right)$ only when $c=i a$ for some $a \in \mathbb{R}^{n}$. Thus, if $u, v \in \mathcal{S}^{\prime}\left(\mathbb{R}^{n}\right)$, cases (iii), (vi) in Theorem 4.6 reduce to case (ii), and case (v) is contained in (iv).

Finally, we discuss the following stability (see $[11,12]$ for related results):

$$
u \circ S-u \otimes v-v \otimes u \in L^{\infty}\left(\mathbb{R}^{2 n}\right),
$$

where $L^{\infty}\left(\mathbb{R}^{2 n}\right)$ denotes the space of bounded measurable functions on $\mathbb{R}^{2 n}$. For the proof we use the following lemma instead of Lemma 4.1.

Lemma 4.8 ([37, p. 122]). Let $f(x, t)$ be a solution of the heat equation. Then $f(x, t)$ satisfies

$$
|f(x, t)| \leq M, \quad x \in \mathbb{R}^{n}, t \in(0,1),
$$

for some $M>0$, if and only if

$$
f(x, t)=\left(f_{0} * E_{t}\right)(x)=\int f_{0}(y) E_{t}(x-y) d y
$$

for some bounded measurable function $f_{0}$ defined in $\mathbb{R}^{n}$. In particular, $f(x, t) \rightarrow$ $f_{0}(x)$ for almost every $x \in \mathbb{R}^{n}$ as $t \rightarrow 0^{+}$.

Following the approach in the proof of Theorem 4.6 we have

$$
|\tilde{u}(x+y, t+s)-\tilde{u}(x, t) \tilde{v}(y, s)-\tilde{v}(x, t) \tilde{u}(y, s)| \leq C,
$$

where $\tilde{u}, \tilde{v}$ are the Gauss transforms of $u, v$. Now, using Corollary 2.7 for $N=0$ and Theorem 4.6 we obtain the following.

Theorem 4.9. Let $u, v \in \mathcal{G}^{\prime}\left(\mathbb{R}^{n}\right)$. Then $(u, v)$ satisfies (4.18) if and only if $(u, v)$ satisfies one of the following:

(i) $u=0$ and $v$ is arbitrary,

(ii) $u$ and $v$ are bounded measurable functions,

(iii) $u=\frac{1}{2 \lambda}\left(e^{c \cdot x}-B(x)\right)$ and $v=\frac{1}{2}\left(e^{c \cdot x}+B(x)\right)$ for some $c \in \mathbb{C}^{n}, \lambda \in \mathbb{C}$ and $B \in L^{\infty}\left(\mathbb{R}^{n}\right)$,

(iv) $u=a \cdot x e^{i c \cdot x}+B(x)$ and $v=e^{i c \cdot x}$ for some $a \in \mathbb{C}^{n}, c \in \mathbb{R}^{n}$ and $B \in L^{\infty}\left(\mathbb{R}^{n}\right)$,

(v) $u=a \cdot x e^{c \cdot x}$ and $v=e^{c \cdot x}$ for some $a, c \in \mathbb{C}^{n}$,

(vi) $u=\frac{1}{2 \lambda}\left(e^{c_{1} \cdot x}-e^{c_{2} \cdot x}\right)$ and $v=\frac{1}{2}\left(e^{c_{1} \cdot x}+e^{c_{2} \cdot x}\right)$ for some $c_{1}, c_{2} \in \mathbb{C}^{n}$ and $\lambda \in \mathbb{C}$.

Let $f$ be a Lebesgue measurable function on $\mathbb{R}^{n}$ satisfying the following condition: for every $\epsilon>0$ there exists $C_{\epsilon}>0$ such that

$$
|f(x)| \leq C_{\epsilon} e^{\epsilon|x|^{2}}
$$


for all $x \in \mathbb{R}^{n}$. The function satisfying (4.20) is said to be an infra-exponential function of order 2 . It is easy to see that every infra-exponential function $f$ of order 2 defines an element of $\mathcal{G}^{\prime}\left(\mathbb{R}^{n}\right)$ via the correspondence

$$
\langle f, \varphi\rangle=\int f(x) \varphi(x) d x
$$

for $\varphi \in \mathcal{G}$. Thus, as a direct consequence of Theorem 4.7 we obtain the following.

Theorem 4.10. Let $f, g$ be infra-exponential functions of order 2. There exists $C>0$ such that

$$
\|f(x+y)-f(x) g(y)-g(x) f(y)\|_{L^{\infty}\left(\mathbb{R}^{2 n}\right)} \leq C
$$

if and only if $f, g$ satisfy one of the following in the almost everywhere sense:

(i) $f=0$ and $g$ is arbitrary,

(ii) $f$ and $g$ are bounded measurable functions,

(iii) $f(x)=\frac{1}{2 \lambda}\left(e^{c \cdot x}-B(x)\right)$ and $g(x)=\frac{1}{2}\left(e^{c \cdot x}+B(x)\right)$ for some $c \in \mathbb{C}^{n}, \lambda \in \mathbb{C}$ and $B \in L^{\infty}\left(\mathbb{R}^{n}\right)$,

(iv) $f(x)=a \cdot x e^{i c \cdot x}+B(x), g(x)=e^{i c \cdot x}$ for some $a \in \mathbb{C}^{n}, c \in \mathbb{R}^{n}$ and $B \in$ $L^{\infty}\left(\mathbb{R}^{n}\right)$,

(v) $f(x)=a \cdot x e^{c \cdot x}$ and $g(x)=e^{c \cdot x}$ for some $a, c \in \mathbb{C}^{n}$,

(vi) $f(x)=\frac{1}{2 \lambda}\left(e^{c_{1} \cdot x}-e^{c_{2} \cdot x}\right)$ and $g(x)=\frac{1}{2}\left(e^{c_{1} \cdot x}+e^{c_{2} \cdot x}\right)$ for some $c_{1}, c_{2} \in \mathbb{C}^{n}$ and $\lambda \in \mathbb{C}$.

\section{Acknowledgements}

The first author was supported by Basic Science Research Program through the National Foundation of Korea (NRF) funded by the Ministry of Education, Science and Technology (MEST) (no. 2012R1A1A008507) and the second author was supported by Basic Science Research Program through the National Foundation of Korea (NRF) funded by the Ministry of Education, Science and Technology (MEST) (no. 2012003264). The authors express their deep thanks to the referee for many valuable comments, prompting indispensable revisions of the paper.

\section{References}

[1] J. Aczél and J. Dhombres, Functional equations in several variables, Cambridge Univ. Press, New York, 1989. Zbl 0685.39006 MR 1004465

[2] T. Aoki, On the stability of the linear transformation in Banach spaces, J. Math. Soc. Japan 2 (1950), 64-66. Zbl 0040.35501 MR 0040580

[3] J. A. Baker, Distributional methods for functional equations, Aequationes Math. 62 (2001), 136-142. Zbl 0989.39009 MR 1849145 
[4] - On a functional equation of Aczél and Chung, Aequationes Math. 46 (1993), 99111. Zbl 0791.39008 MR 1220725

[5] - Functional equations, tempered distributions, and Fourier transforms, Trans. Amer. Math. Soc. 315 (1989), 57-68. Zbl 0674.39010 MR 0979965

[6] D. G. Bourgin, Classes of transformations and bordering transformations, Bull. Amer. Math. Soc. 57 (1951), 223-237. Zbl 0043.32902 MR 0042613

[7] Multiplicative transformations, Proc. Nat. Acad. Sci. U.S.A. 36 (1950), 564-570. Zbl 0039.33403 MR 0038569

[8] J. Chung, Stability of exponential equations in Schwartz distributions, Nonlinear Anal. 69 (2008), 3503-3511. Zbl 1157.39015 MR 2450555

[9] Stability of approximately quadratic Schwartz distributions, Nonlinear Anal. 67 (2007), 175-186. Zbl 1116.39017 MR 2313887

[10] J. Chung and J. Chang, On the stability of trigonometric functional equations in distributions and hyperfunctions, Abstract Appl. Anal. 2013, art. ID 275915, 12 pp. Zbl 1275.39014 MR 3055863

[11] Stability of trigonometric functional equations in generalized functions, J. Inequal. Appl. 2010, art. ID 801502, 12 pp. Zbl 1187.39034 MR 2592856

[12] The stability of the sine and cosine functional equations in Schwartz distributions, Bull. Korean Math. Soc. 45 (2009), 87-97. Zbl 1175.39015 MR 2488504

[13] J. Chung, S.-Y. Chung and D. Kim, A characterization for Fourier hyperfunctions, Publ. RIMS Kyoto Univ. 30 (1994), 203-208. Zbl 0808.46056 MR 1265470

[14] Une caractérisation de l'espace de Schwartz, C. R. Acad. Sci. Paris Sér. I Math. 316 (1993), 23-25. Zbl 0820.46033 MR 1198743

[15] S.-Y. Chung, An heat equation approach to distributions with $L^{p}$ growth, Comm. Korean Math. Soc. 9 (1994), 897-903.

[16] S.-Y. Chung, D. Kim and E. G. Lee, Periodic hyperfunctions and Fourier series, Proc. Amer. Math. Soc. 128 (2000), 2421-2430. Zbl 0951.46016 MR 1657782

[17] S. Czerwik, Stability of functional equations of Ulam-Hyers-Rassias type, Hadronic Press, Palm Harbor, FL, 2003.

[18] I. Fenyö, On an inequality of P. W. Cholewa, in General inequalities, 5 (Oberwolfach, 1986), Int. Schriftenreihe Numer. Math. 80, Birkhäuser, 1987, 277-280. Zbl 0636.39006 MR 1018153

[19] G. L. Forti, Hyer-Ulam stability of functional equations in several variables, Aequationes Math. 50 (1995), 143-190. Zbl 0836.39007 MR 1336866

[20] I. M. Gelfand and G. E. Shilov, Generalized functions II, Academic Press, New York, 1968. Zbl 0159.18301 MR 0435832

[21] _ Generalized functions IV, Academic Press, New York, 1968. MR 0435834

[22] L. Hörmander, The analysis of linear partial differential operators I, Springer, Berlin, 1983. Zbl 0521.35001 MR 0717035

[23] D. H. Hyers, On the stability of the linear functional equations, Proc. Nat. Acad. Sci. U.S.A. 27 (1941), 222-224. Zbl 0061.26403 MR 0004076

[24] D. H. Hyers, G. Isac and Th. M. Rassias, Stability of functional equations in several variables, Birkhäuser, 1998. Zbl 0907.39025 MR 1639801

[25] S. M. Jung, Hyers-Ulam-Rassias stability of functional equations in nonlinear analysis, Springer, New York, 2011. Zbl 1221.39038 MR 2790773

[26] T. Matsuzawa, A calculus approach to hyperfunctions II, Trans. Amer. Math. Soc. 313 (1989), 619-654. Zbl 0681.46042 MR 0997676 
[27] J. M. Rassias, On approximation of approximately linear mappings by linear mappings, J. Funct. Anal. 46 (1982), 126-130. Zbl 0482.47033 MR 0654469

[28] - On approximation of approximately linear mappings by linear mappings, Bull. Sci. Math. 108 (1984), 445-446. Zbl 0599.47106 MR 0784679

[29] Solution of a problem of Ulam, J. Approx. Theory $\mathbf{5 7}$ (1989), 268-273. Zbl 0672.41027 MR 0999861

[30] Th. M. Rassias, On the stability of functional equations in Banach spaces, J. Math. Anal. Appl. 251 (2000), 264-284. Zbl 0964.39026 MR 1790409

[31] On the stability of the linear mapping in Banach spaces, Proc. Amer. Math. Soc. 7 (1978), 297-300. Zbl 0398.47040 MR 0507327

[32] K. Ravi and M. Arunkumar, On the Ulam-Gavruta-Rassias stability of the orthogonally Euler-Lagrange type functional equation, Int. J. Appl. Math. Statist. 7 (2007), 143-156. MR 2339517

[33] L. Schwartz, Théorie des distributions, Hermann, Paris, 1966. Z Zbl 0149.09501 MR 0209834

[34] L. Székelyhidi, The stability of sine and cosine functional equations, Proc. Amer. Math. Soc. 110 (1990), 109-115. Zbl 0718.39004 MR 1015685

[35] The stability of d'Alembert-type functional equations, Acta Sci. Math. (Szeged) 44 (1982), 313-320. Zbl 0517.39008 MR 0684388

[36] S. M. Ulam, Problems in modern mathematics, Chapter VI, Wiley, New York, 1964. Zbl 0137.24201 MR 0280310

[37] D. V. Widder, The heat equation, Academic Press, New York, 1975. Zbl 0322.35041 MR 0466967 